\title{
Numerical Estimation of Traveling Wave Solution of Two-Dimensional K-dV Equation Using a New Auxiliary Equation Method
}

\author{
Rezaul Karim', Abdul Alim², Laek Sazzad Andallah ${ }^{3}$ \\ ${ }^{1}$ Department of Mathematics, Jagannath University, Dhaka, Bangladesh \\ ${ }^{2}$ Department of Mathematics, BUET, Dhaka, Bangladesh \\ ${ }^{3}$ Department of Mathematics, Jahangirnagar University, Savar, Bangladesh \\ Email:rrezaul@yahoo.com,maalim@math.buet.ac.bd, andallahls@gmail.com
}

Received November 12, 2012; revised December 17, 2012; accepted December 30, 2012

\begin{abstract}
Korteweg de-Vries (K-dV) has wide applications in physics, engineering and fluid mechanics. In this the Korteweg deVries equation with traveling solitary waves and numerical estimation of analytic solutions have been studied. We have found some exact traveling wave solutions with relevant physical parameters using new auxiliary equation method introduced by PANG, BIAN and CHAO. We have solved the set of exact traveling wave solution analytically. Some numerical results of time dependent wave solutions have been presented graphically and discussed. This procedure has a potential to be used in more complex system of many types of K-dV equation.
\end{abstract}

Keywords: Contor; Propagation; Soliton Periodic; Time Evolution

\section{Introduction}

Yu. N. Zaiko studied the presence of a singularity results in that the velocity of long wave perturbations in the system becomes imaginary, which corresponds to the wave propagation in the range of nontransparency [1]. Qu Fuli and Wang Wenqia studied linear unconditionally stable for nonlinear third-order $\mathrm{K}-\mathrm{dV}$ equation by the analysis of linearization procedure and is used directly on the parallel computer [2]. Rheir numerical experiment shows that their method has high accuracy. A model of an income- pressible flow through a cylindrical metal pipe and the fundamental physical and mathematical facts presented in $[3,4]$ are used to show how a solitary velocity wave (solution) can arise in this system, V. A. Rukavishnikov \& O. P. Tkachenko are studied [5]. Although the resulting asymptotic expressions in the radial coordinate differ considerably from the classical expansion in depth for shallow-water waves, they are able to derive the Kd-V equation. They also show how to proceed back from the Kd-V equation to the velocity function and present the numerical results obtained for a model problem. Nejib Smaoui and Rasha H. Al-Jamal studied the boundary control problem of the generalized Korteweg-de Vries Burger (GKdVB) equation on the interval $[0,1]$, [6]. They presented a numerical results supporting the analytical ones for both the controlled and uncontrolled equations using a finite element method.
Jing Pang et al. studied the method of finding the traveling wave solution to K-dV equation using a new auxiliary equation method [7]. They got a set of traveling wave solution for a specific $3^{\text {rd }}$-order $\mathrm{K}-\mathrm{dV}$ equation. We studied the governing two-dimensional $3^{\text {rd }}$ order K-dV equation. After some suitable transformation we got a simple form of K-dV Equation (8). Using a new auxiliary equation method we got the ten sets of travelling wave solution of $\mathrm{K}-\mathrm{dV}$ equation for real case. There are three cases to be arisen in our study. Two of them are real sense and the other is imaginary concept. After getting the analytical solution of K-dV equation we discussed the ten sets of traveling wave solution numerically and their physical phenomena are described in result and discussion section.

\section{Method of Solution}

The remarkable form of Korteweg-de Vries nonlinear partial differentiable equation [8] is:

$$
u_{t}+C_{0}\left(1+\frac{3 u}{2 h_{0}}\right) u_{x}+\sigma u_{x x x}=0
$$

which was first introduced by Dutch mathematics Diederik Korteweg and Gustav de Vries in 1895, to describe long water waves in a channel of depth $h_{0}$, where $\sigma=\frac{1}{6} c_{0} h_{0}^{2}$ is a constant for fairly long waves, 
$c_{0}=\left(g h_{0}\right)^{\frac{1}{2}}, u$ is displacement of wave and $g$ is the acceleration due to gravity. In this section we introduce the method of finding the analytic wave solution to nonlinear evolution equation due to Jing Pang, Chun-quan Bian, and Lu Chao [7]. First, a given nonlinear partial differential equation has the form.

$$
p\left(u, u_{t}, u_{x}, u_{t t}, u_{x x}, \cdots\right)=0
$$

This method mainly consists of four steps:

\subsection{Step 1}

Take the complex solutions of (1) in the form

$$
u(x, t)=u(\xi), \xi=x-u t
$$

where $u$ is a real constant. Under the transformation (2), (1) becomes an ordinary differential equation

$$
Q\left(u, u^{\prime}, u^{\prime \prime}, \cdots\right)=0
$$

\subsection{Step 2}

Take the solutions of (3) in the more general form:

$$
u(\xi)=a_{0}+\sum_{i=1}^{m} a_{i}\left(\frac{G(\xi)}{G^{\prime}(\xi)}\right)^{i}+b_{i}\left(\frac{G(\xi)}{G^{\prime}(\xi)}\right)^{-i}
$$

where $a_{m}$ and $b_{m}$ are not zero at the same time, and $a_{0}, a_{i}$ and $b_{i}(i=1,2,3, \cdots, m)$ are constants to be determined later. The integer $m$ in (4) can be determined by balancing the highest order nonlinear terms and the highest order linear terms of $u(\xi)$ in (3). $G=G(\xi)$ satisfies the second-order linear ordinary differential equation

$$
G^{\prime \prime}+\lambda G^{\prime}+\mu G=0
$$

where $\lambda$ and $\mu$ are constants for the general solution of (5) as follows:

When $\lambda^{2}-4 \mu>0$,

$$
\begin{aligned}
G(\xi)= & c_{1} \exp \left(\frac{-\lambda+\sqrt{\lambda^{2}-4 \mu}}{2} \xi\right) \\
& +c_{1} \exp \left(\frac{-\lambda-\sqrt{\lambda^{2}-4 \mu}}{2} \xi\right) ;
\end{aligned}
$$

When $\lambda^{2}-4 \mu=0, G(\xi)=\left(c_{1}+c_{2} \xi\right) \exp \left(-\frac{\lambda}{2} \xi\right)$;

When $\lambda^{2}-4 \mu<0$,

$$
\begin{aligned}
G(\xi)= & \exp \left(-\frac{\lambda}{2} \xi\right)\left(c_{1} \cos \frac{\sqrt{4 \mu-\lambda^{2}}}{2} \xi\right. \\
& \left.+c_{2} \sin \frac{\sqrt{4 \mu-\lambda^{2}}}{2} \xi\right)
\end{aligned}
$$

Note: Let $a_{i}=0, i=1,2, \cdots, m$. Equation (4) changes to

$$
u(\xi)=a_{0}+\sum_{i=1}^{m} b_{i}\left(\frac{G(\xi)}{G^{\prime}(\xi)}\right)^{-i}
$$

The form of (6) has been used in (Deng, Z. G., et al., 2009). If we set $b_{i}=0(i=1,2, \cdots, m)$, (4) changes to

$$
u(\xi)=a_{0}+\sum_{i=1}^{m} a_{i}\left(\frac{G(\xi)}{G^{\prime}(\xi)}\right)^{i}
$$

\subsection{Step 3}

Substitute (4) into (3) and collect all terms with the same order of $\frac{G}{G^{\prime}}$ together. The left-hand side of (3) is converted into a polynomial in $\frac{G}{G^{\prime}}$. Then, let each coefficient of this polynomial to be zero to derive a set of over-determined partial differential equations for $a_{0}, a_{i}, b_{i}(i=1,2, \cdots, m), \lambda, \mu$, and $v$.

\subsection{Step 4}

Solve the algebraic equations obtained in step 3 with the aid of a computer algebra system (such as Mathematica or Maple) to determine these constants. Moreover, the solutions of (5) are well known. Then substituting $a_{0}$, $a_{i}, b_{i}(i=1,2, \cdots, m), v$, and the solutions of (5) into (4), we can obtain the exact analytical/traveling wave solutions of (1).

\section{Solution of Mathematical Problem}

Consider the $\mathrm{K}-\mathrm{dV}$ equation

$$
u_{t}+u u_{x}+u_{x x x}=0
$$

describe the evolution of long wave (with large length and measurable amplitude) down a canal with a rectangular cross section. Here $u$ represents the wave amplitude, and $u_{t}$ represents the vertical velocity of the wave at ( $x$, $t), u_{x}$ describes the rate of change in amplitude with respect to $x$ and $u_{x x x}$ is a dispersion term. This means that if $u$ is the amplitude of wave at some point in space, then $u_{x}$ is the slope of the wave at the point and $u_{x x}$ concavity near the point as given in $[9,10]$. The existence of solitary waves is due to the balancing effects of $u u_{x}$ and $u_{x x x}$ in Equation (8). The nonlinear term $u u_{x}$ in Equation (8) is important because the amplitude of the wave depends on its own rate of change in space; it also represents steepening. The term $u_{x x x}$ implies dispersion of different frequency components.

Now we choose the traveling wave transformation (2) i.e. $u(x, t)=u(\xi), \quad \xi=x-v t$ where $v=$ constant. 
Substituting these into (8), integrating it with respect to $\xi$ once, and letting the integrating constant to be zero, we have

$$
u_{\xi \xi}+\frac{1}{2} u^{2}-v u=0
$$

According to step 2 we get $m=2$. Therefore we can write the solution of (9) in the form

$$
u(\xi)=a_{0}+\sum_{i=1}^{2} a_{i}\left(\frac{G(\xi)}{G^{\prime}(\xi)}\right)^{i}+b_{i}\left(\frac{G(\xi)}{G^{\prime}(\xi)}\right)^{i}
$$

That is

$$
u(\xi)=a_{0}+a_{1} \frac{G}{G^{\prime}}+a_{2}\left(\frac{G}{G^{\prime}}\right)^{2}+b_{1}\left(\frac{G}{G^{\prime}}\right)^{-1}+b_{2}\left(\frac{G}{G^{\prime}}\right)^{-2}
$$

where $a_{2}$ and $b_{2}$ are not zero at he same time. By using (5) and from (10), we have

$$
\begin{aligned}
u^{\prime \prime}(\xi)= & \left(\lambda a_{1}+2 a_{2}+\lambda \mu b_{1}+2 \mu^{2} b_{2}\right) \\
& +\left(\lambda^{2} a_{1}+6 \lambda a_{2}+2 \mu a_{1}\right)\left(\frac{G}{G^{\prime}}\right) \\
& +\left(8 \mu a_{2}+3 \lambda \mu a_{1}+4 \lambda^{2} a_{2}\right)\left(\frac{G}{G^{\prime}}\right)^{2} \\
& +\left(2 \mu^{2} a_{1}+10 \lambda \mu a_{2}\right)\left(\frac{G}{G^{\prime}}\right)^{3}+6 \mu^{2} a_{2}\left(\frac{G}{G^{\prime}}\right)^{4} \\
& +\left(2 \mu b_{1}+6 \lambda \mu b_{2}+\lambda^{2} b_{1}\right)\left(\frac{G}{G^{\prime}}\right)^{-1} \\
& +\left(3 \lambda b_{1}+4 \lambda^{2} b_{2}+8 \mu b_{2}\right)\left(\frac{G}{D^{\prime}}\right)^{-2} \\
& +\left(2 b_{1}+10 \lambda b_{2}\right)\left(\frac{G}{G^{\prime}}\right)^{-3}+6 b_{2}\left(\frac{G}{G^{\prime}}\right)^{-4}
\end{aligned}
$$

Substituting (10) and (11) into (9) and collecting the coefficients of $\left(\frac{G}{G^{\prime}}\right)(i=0, \pm 1, \pm 2, \pm 3, \pm 4)$, and letting it be zero without loss of generality we obtain the system:

$$
\begin{gathered}
\lambda a_{1}+2 a_{2}+\lambda \mu b_{1}+2 \mu^{2} b_{2}+\frac{1}{2} a_{0}^{2}+a_{1} b_{1}+a_{2} b_{2}-v a_{0}=0 \\
\lambda^{2} a_{1}+6 \lambda a_{2}+2 \mu a_{1}+a_{0} a_{1}+a_{2} b_{1}-v a_{1}=0 \\
8 \mu a_{2}+3 \lambda \mu a_{1}+4 \lambda^{2} a_{2}+\frac{1}{2} a_{1}^{2}+a_{0} a_{2}-v a_{2}=0 \\
2 \mu^{2} a_{1}+10 \lambda \mu a_{2}+a_{1} a_{2}=0 \\
6 \mu^{2} a_{2}+\frac{1}{2} a_{2}^{2}=0 \\
2 \mu b_{1}+6 \lambda \mu b_{2}+\lambda^{2} b_{1}+a_{0} b_{1}+a_{1} b_{2}-v b_{1}=0 \\
3 \lambda b_{1}+4 \lambda^{2} b_{2}+8 \mu b_{2}+\frac{1}{2} b_{1}^{2}+a_{0} b_{2}-v b_{2}=0
\end{gathered}
$$

$$
\begin{gathered}
2 b_{1}+10 \lambda b_{2}+b_{1} b_{2}=0 \\
6 b_{2}+\frac{1}{2} b_{2}^{2}=0
\end{gathered}
$$

From (ix) we get either $b_{2}=0$ or $b_{2}=-12$ and from (v) either $a_{2}=0$ or $a_{2}=-12 \mu^{2}$. So, there are three cases to be arisen. For $b_{2}=0$ and $a_{2}=0$ uses the system of equations (i) to (ix) we get trivial solutions.

Trivial solution set is $a_{0}=a_{1}=a_{2}=b_{1}=b_{2}=0$ and the other solution sets are as follows:

For $a_{2}=-12 \mu^{2}$ and $b_{2}=0$, using the system of Equations (i)-(ix) we get a set of solution is as follows:

$$
\begin{gathered}
a_{0}=0, a_{1}=-12 \lambda \mu, a_{2}=-12 \mu^{2}, \\
b_{1}=0, b_{2}=0, v=\lambda^{2}+8 \mu
\end{gathered}
$$

For $a_{2}=0$ and $b_{2}=-12$, using the system of Equations (i)-(ix) we get a set of solutions are as follows:

$$
\begin{gathered}
a_{0}=-12 \mu, a_{1}=0, a_{2}=0, \\
b_{1}=-12 \lambda, b_{2}=-12, v=\lambda^{2}-4 \mu \\
a_{0}=-2 \lambda^{2}-4 \mu, a_{1}=0, a_{2}=0, \\
b_{1}=-12 \lambda, b_{2}=-12, v=-\lambda^{2}+4 \mu
\end{gathered}
$$

For $a_{2}=-12 \mu^{2}$ and $b_{2}=-12$, using the system of Equations (i)-(ix) we get a set of solutions are as follows:

$$
\begin{gathered}
a_{0}=8 \mu, a_{1}=0, a_{2}=-12 \mu^{2}, \\
b_{1}=0, b_{2}=-12, v=16 \mu, \lambda=0
\end{gathered}
$$

and

$$
\begin{gathered}
a_{0}=-24 \mu, a_{1}=0, a_{2}=-12 \mu^{2}, \\
b_{1}=0, b_{2}=-12, v=-16 \mu, \lambda=0
\end{gathered}
$$

where $\lambda$ and $\mu$ are arbitrary constants. By using (A)(E) Equation (10) can be written as:

$$
u(\xi)=a_{0}+a_{1} \frac{G}{G^{\prime}}+a_{2}\left(\frac{G}{G^{\prime}}\right)^{2}+b_{1}\left(\frac{G}{G^{\prime}}\right)^{-1}+b_{2}\left(\frac{G}{G^{\prime}}\right)^{-2}
$$

Equations (A)-(E) and (10) implies respectively as follows:

$$
\begin{gathered}
u(\xi)=-12 \lambda \mu\left(\frac{G}{G^{\prime}}\right)-12 \mu^{2}\left(\frac{G}{G^{\prime}}\right)^{2}, \\
\xi=x-\left(\lambda^{2}+8 \mu\right) t \\
u(\xi)=-12 \mu-12 \lambda\left(\frac{G}{G^{\prime}}\right)^{-1}-12\left(\frac{G}{G^{\prime}}\right)^{-2}, \\
\xi=x-\left(\lambda^{2}-4 \mu\right) t \\
u(\xi)=-2 \lambda^{2}-4 \mu-12 \lambda\left(\frac{G}{G^{\prime}}\right)^{-1}-12\left(\frac{G}{G^{\prime}}\right)^{-2}, \\
\xi=x+\left(\lambda^{2}-4 \mu\right) t
\end{gathered}
$$




$$
\begin{gathered}
u(\xi)=8 \mu-12 \mu^{2}\left(\frac{G}{G^{\prime}}\right)^{2}-12\left(\frac{G}{G^{\prime}}\right)^{-2}, \\
\xi=x-16 \mu t, \lambda=0 \\
u(\xi)=-24 \mu-12 \mu^{2}\left(\frac{G}{G^{\prime}}\right)^{2}-12\left(\frac{G}{G^{\prime}}\right)^{-2} ; \\
\xi=x+16 \mu t, \lambda=0
\end{gathered}
$$

Now, the second order differential Equation (5) is as follows:

$$
G^{\prime \prime}+\lambda G^{\prime}+\mu G=0
$$

When, $\lambda^{2}-4 \mu>0$,

$$
\begin{aligned}
G(\xi)= & c_{1} \exp \left(\frac{-\lambda+\sqrt{\lambda^{2}-4 \mu}}{2} \xi\right) \\
& +c_{2} \exp \left(\frac{-\lambda-\sqrt{\lambda^{2}-4 \mu}}{2} \xi\right) ;
\end{aligned}
$$

When $\lambda^{2}-4 \mu=0, G(\xi)=\left(c_{1}+c_{2} \xi\right) \exp \left(-\frac{\lambda}{2} \xi\right)$;

When $\lambda^{2}-4 \mu<0$,

$$
\begin{aligned}
G(\xi)= & \exp \left(-\frac{\lambda}{2} \xi\right)\left(c_{1} \cos \frac{\sqrt{4 \mu-\lambda^{2}}}{2} \xi\right. \\
& \left.+c_{2} \sin \frac{\sqrt{4 \mu-\lambda^{2}}}{2} \xi\right)
\end{aligned}
$$

\subsection{Case I}

For the first case: $\lambda^{2}-4 \mu>0$

$$
\frac{G}{G^{\prime}}=\frac{2\left(1+c \mathrm{e}^{-M \xi}\right)}{(-\lambda+M)-(\lambda+M) c \mathrm{e}^{-M \xi}}
$$

where, $M=\sqrt{\lambda^{2}-4 \mu}$

Therefore, Equations (F)-(J) becomes:

$$
\begin{aligned}
u(\xi)= & -24 \lambda \mu\left[\frac{1+c \mathrm{e}^{-M \xi}}{(-\lambda+M)-(\lambda+M) c \mathrm{e}^{-M \xi}}\right] \\
& -24 \mu^{2}\left[\frac{1+c \mathrm{e}^{-M \xi}}{(-\lambda+M)-(\lambda+M) c \mathrm{e}^{-M \xi}}\right]
\end{aligned}
$$

where $\xi=x-\left(\lambda^{2}+8 \mu\right) t, M=\sqrt{\lambda^{2}-4 \mu}$

$$
\begin{aligned}
u(\xi)= & -24 \lambda \mu\left[\frac{1+c \mathrm{e}^{-M \xi}}{(-\lambda+M)-(\lambda+M) c \mathrm{e}^{-M \xi}}\right] \\
& -24 \mu^{2}\left[\frac{1+c \mathrm{e}^{-M \xi}}{(-\lambda+M)-(\lambda+M) c \mathrm{e}^{-M \xi}}\right]
\end{aligned}
$$

where $\xi=x-\left(\lambda^{2}+8 \mu\right) t, M=\sqrt{\lambda^{2}-4 \mu}$

$$
\begin{aligned}
& u(\xi)=-12 \mu-6 \lambda\left[\frac{(-\lambda+M)-(\lambda+M) c \mathrm{e}^{-M \xi}}{1+c \mathrm{e}^{-M \xi}}\right] \\
& -3\left[\frac{(-\lambda+M)-(\lambda+M) c \mathrm{e}^{-M \xi}}{1+c \mathrm{e}^{-M \xi}}\right]^{2} \\
& \xi=x-\left(\lambda^{2}-4 \mu\right) t, M=\sqrt{\lambda^{2}-4 \mu} \\
& u(\xi)=-2 \lambda^{2}-4 \mu-6 \lambda\left[\frac{(-\lambda+M)-(\lambda+M) c \mathrm{e}^{-M \xi}}{1+c \mathrm{e}^{-M \xi}}\right] \\
& -3\left[\frac{(-\lambda+M)-(\lambda+M) c \mathrm{e}^{-M \xi}}{1+c \mathrm{e}^{-M \xi}}\right]^{2}, \\
& \xi=x+\left(\lambda^{2}-4 \mu\right) t, M=\sqrt{\lambda^{2}-4 \mu} \\
& u(\xi)=8 \mu-48 \mu^{2}\left[\frac{1+c \mathrm{e}^{-M \xi}}{(-\lambda+M)-(\lambda+M) c \mathrm{e}^{-M \xi}}\right]^{2} \\
& -3\left[\frac{1+c \mathrm{e}^{-M \xi}}{(-\lambda+M)-(\lambda+M) c \mathrm{e}^{-M \xi}}\right]^{2} \\
& \xi=x-16 \mu t, M=\sqrt{\lambda^{2}-4 \mu}, \lambda=0 \\
& u(\xi)=-24 \mu-48 \mu^{2}\left[\frac{1+c \mathrm{e}^{-M \xi}}{(-\lambda+M)-(\lambda+M) c \mathrm{e}^{-M \xi}}\right]^{2} \\
& -3\left[\frac{1+c \mathrm{e}^{-M \xi}}{(-\lambda+M)-(\lambda+M) c \mathrm{e}^{-M \xi}}\right]^{2}, \\
& \xi=x+16 \mu t, M=\sqrt{\lambda^{2}-4 \mu}, \lambda=0
\end{aligned}
$$

\subsection{Results and Discussion}

We study here the interaction of two wave solutions to the third order K-dV equation. The numerical representation of two dimensional third order $\mathrm{K}-\mathrm{dV}$ equations for this problem are obtained by the Fortran scheme and compared with analytical solution cases for $\lambda=5.0$, $\mu=1.25$, and $c=10.0$ such that $\lambda^{2}-4 \mu \succ 0$. Numerical representation generates that the same behavior as wave solutions. The solutions remain unchanged before and after their interaction. As seen in Figure 1(a) in Equation (12), time evolution of $u$ wave for different values of displacement on the domain $[0,1]$. Here the $u$ wave varies with displacement. It is found that the water flow oscillates regularly that is periodic over the displacement region $50 \leq x \leq 250$. In Figure 1(b), $u$ wave for different values of time $t$ against for the whole region of displacement $0 \leq x \leq 50$ and time $0.2 \leq t \leq 0.1$. It is seen that the wave increases gradually as time increases. Figure 2 describes the phase $u(\xi)$ from the equation 12 with respect to time and displacement. It shows an 


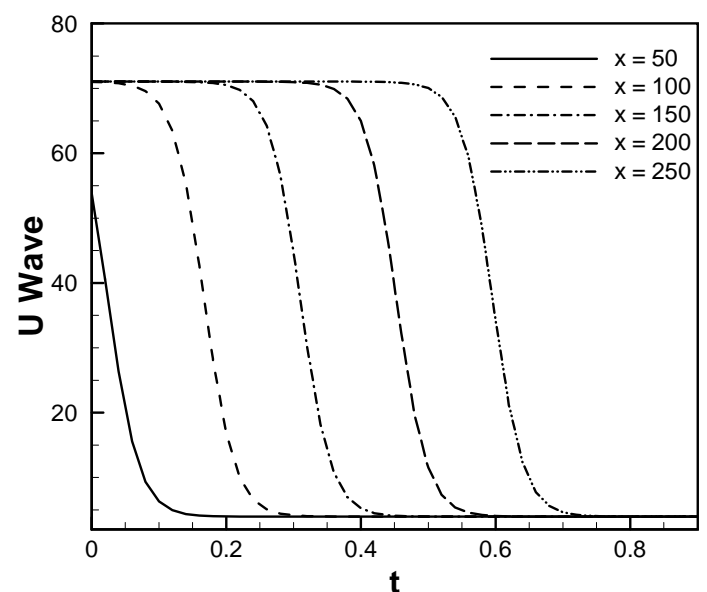

(a)

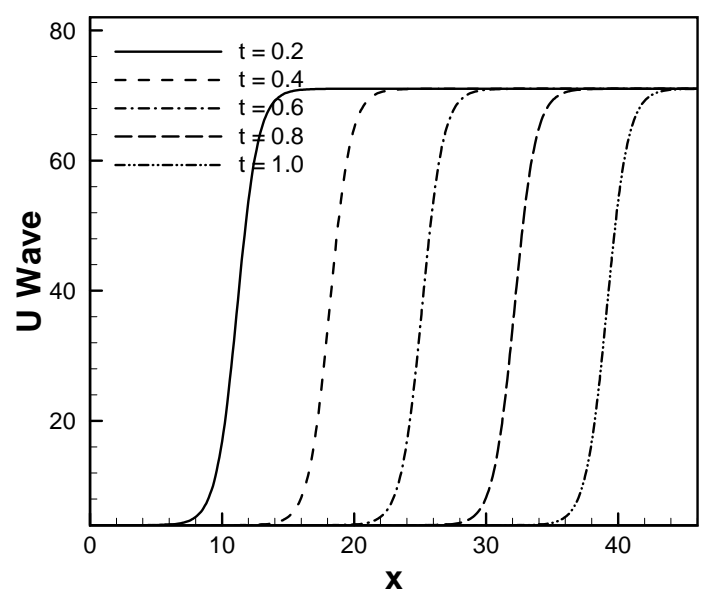

(b)

Figure 1. $u$ wave (a) against $t$ for different values of $x$ (b) against $x$ for different values of $t$.

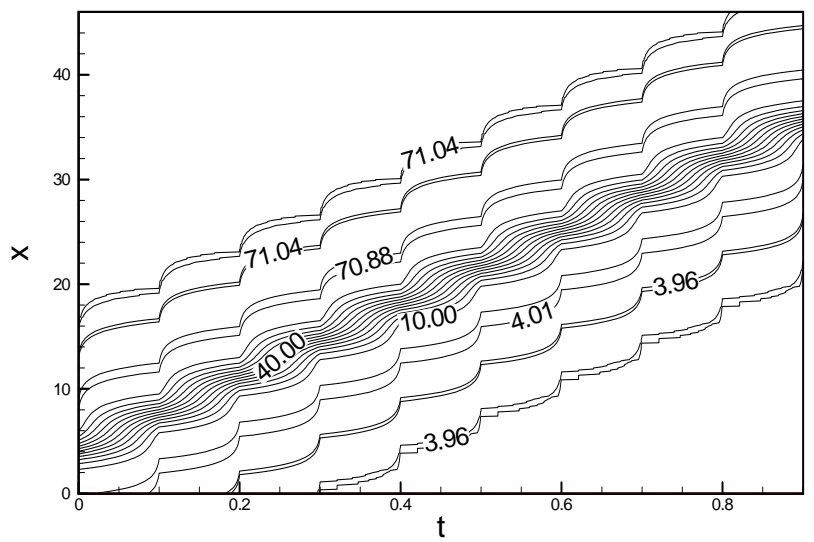

Figure 2. $u$ wave contour against $t$ and $x$.

example of two solution on the domain $[0,40] \times[0,1]$. As expected, we see that the soliton travels until they collide, but their amplitudes are unchanged and position in time has changed considerably. Amplitude of $u$ wave with respect to $t$ and $x$ gradually increases smoothly. In
Figure 3(a) for the wave solution of $\mathrm{K}-\mathrm{dV}$ equation represented by 13, time evolution of $u$ wave against $t$ for different values of $x$. The graphical representation of Equation (13) we consider the constants $\lambda=5.0, \mu=1.25$, and $c=10.0$ such that we choose the values for $\lambda^{2}-4 \mu \succ 0$ but $c$ does not depend on $\lambda$ and $\mu$. It is found that the water flow oscillates regularly that is periodic for $x=5.0, x=10.0, x=15.0, x=20.0$ and $x=$ 25.0 against $t$. Here the time $t$ defined over the interval [0, 1.6]. Figure 3(b) describes $u$ wave for different values of time $t$ against $x$. Here $x$ varies from 0 to 50 for $t=0.1, t$ $=0.5, t=0.9, t=1.3$ and $t=1.7$. As seen in Figure 4, $u$ wave contour against $t$ and $x$. For $0 \leq t \leq 2$ and $0 \leq x \leq 40$, the contour gradually increases and it is started from -284.164 and finishes -15.8359 . As expected, we see that the solitons travel until they collide and the wave gradually increases. Figure 5(a) we found the $u$ wave against $t$ for different values of $x$ while the constants $\lambda=$ 5.0, $\mu=1.25$, and $c=10.0$ so that $\lambda^{2}-4 \mu \succ 0$. The graphical representation of equation 14, water wave $u$ is increased before the collision. In this case $u$ wave is define over the interval $-1.25 \leq t \leq 0$ for $x=5.0, x=10.0$,

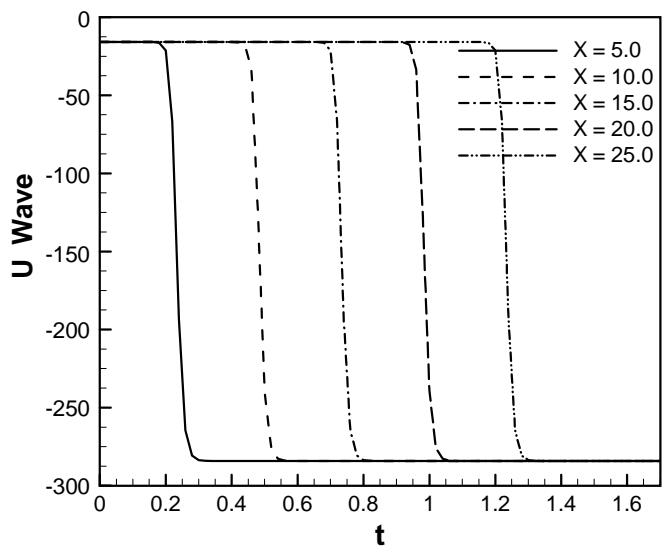

(a)

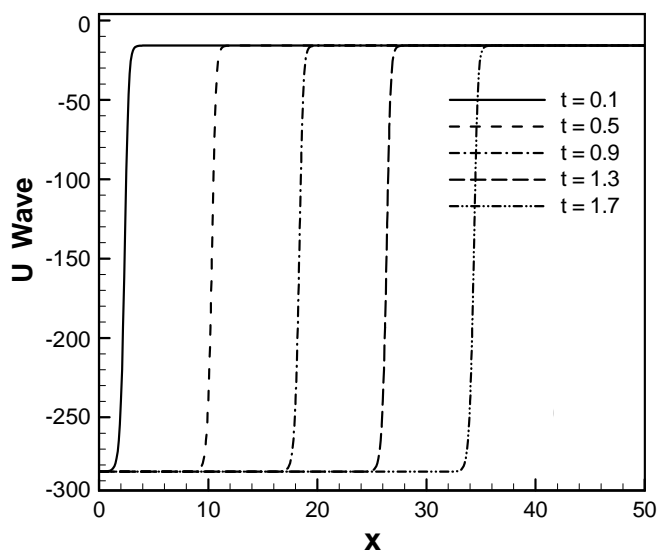

(b)

Figure 3. $u$ wave (a) against $t$ for different values of $x$ (b) against $x$ for different values of $t$. 


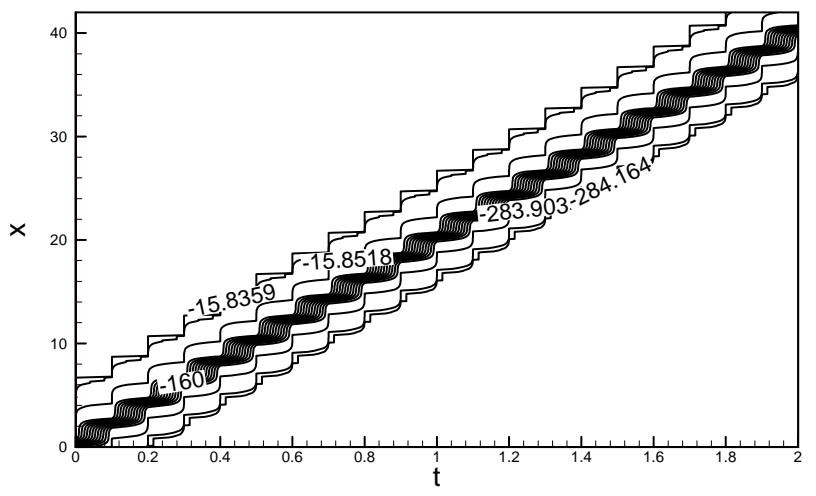

Figure 4. $u$ wave contour against $t$ and $x$.

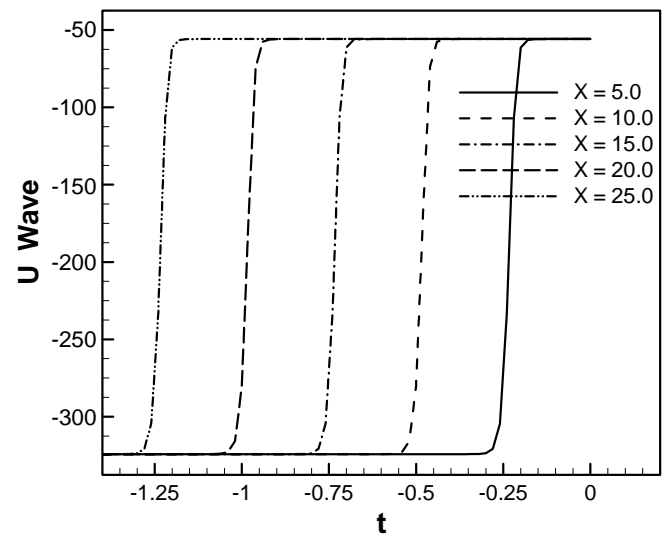

(a)

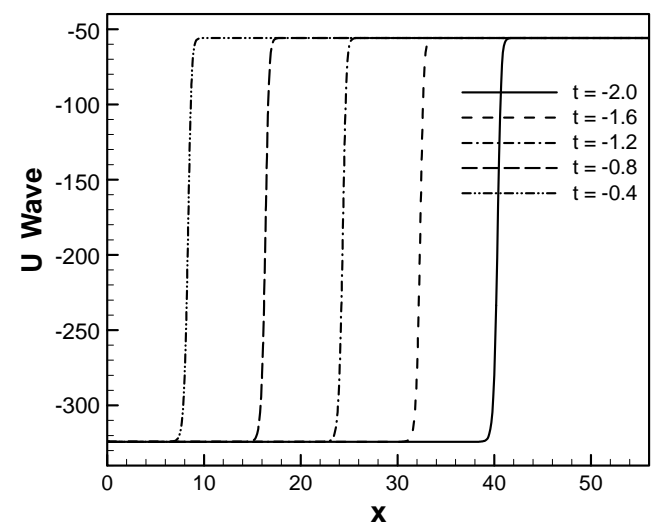

(b)

Figure 5. $u$ wave (a) against $t$ for different values of $x$ (b) against $\boldsymbol{x}$ for different values of $\boldsymbol{t}$.

$x=15.0, x=20.0$ and $x=25.0$. Figure 5(b) describes $u$ wave increases against $x$ for $t=-2.0, t=-1.6, t=-1.2, t$ $=-0.8$ and $t=0.4$. Interesting things are to be found in both cases, $u$ wave gradually increases against $t$ for different values of $x$ and against $x$ for different values of $t$ before collision. As seen in Figure 6, $u$ wave contour against $t$ and $x$. For the both cases we plot the phase $u(\xi)$ for the Equation (14) with respect to time and displacement. As expected, we see that $u$ wave contour gradually increases like $-324.161,-160,-55.8359$ and so on over the domain $[0,40] \times[-2,0]$. Next, contours of typical flow patterns are seen in Figure 7(a) which is based on equation 15 . The branch is composed of associated over $0 \leq t \leq 2$ and displacement $5 \leq x \leq 25$. It is found that the flow oscillates irregularly that is multiperiodic oscillation. We are also interested in trying our scheme for the wave solution of the $\mathrm{K}-\mathrm{dV}$ equation. In

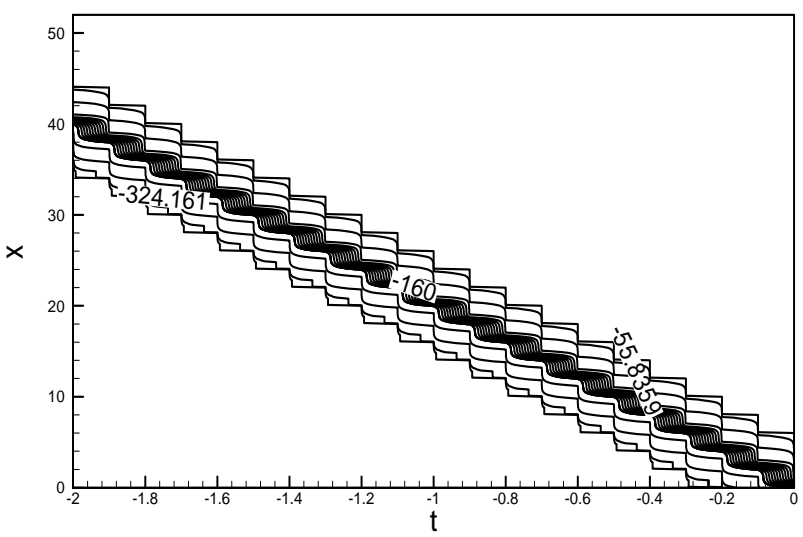

Figure 6. $u$ wave contour against $t$ and $x$.

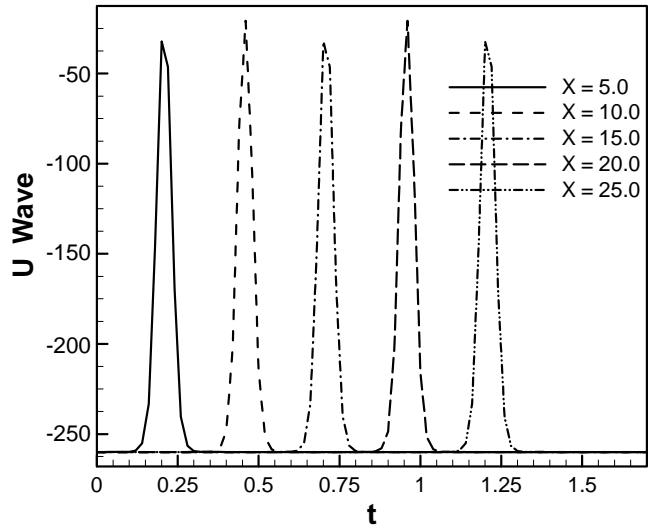

(a)

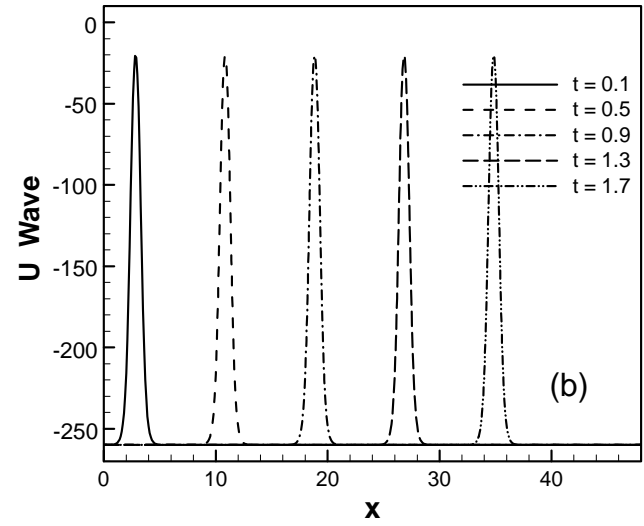

(b)

Figure 7. $u$ wave (a) against $t$ for different values of $x$ (b) against $x$ for different values of $t$. 
this figure we see that the behavior of the $u$ wave is exactly what we expect in the analytical sense, the only thing that the change about the wave once in time for $x=$ 5.0, $x=10.0, x=15.0, x=20.0$ and $x=25.0$ anywhere else about the wave stays the same. In Figure 7(b) we see that wave solution follows that the exact solution fairly closely with respect to displacement over $t=0.1, t$ $=0.5, t=0.9, t=1.3$ and $t=1.7$. The general behavior of wave solution of $\mathrm{K}-\mathrm{dV}$ equation remains same however. Figure 8 describes $u$ wave contour against $t$ and $x$ over the domain $[0,40] \times[0,2]$. $U$ wave contour is maximum on the top of the wave but diminishes from the top to both the sides of contour. Here, we see that at the top the value of the contour -100 and it gradually decreases to the both sides like -260 to the left and -260 to the right. The plot of function (16) is shown in Figure 9(a) we have found $u$ wave against $t$ for different values of $x$. In Figure 9(b) indicates that the $u$ wave against $x$ for different values of $t$. Here we have seen that the $u$ wave against $x$ for the different values of $t$ like $t=-2.0, t$ $=-1.6, t=-1.2, t=-0.8$ and $t=-0.4$, are exact to the previous one. For the both cases it shows that the waves are running against $t$ and $x$ respectively. Figure 10 describes $u$ wave contour against $t$ and $x$ over the domain $[0,25] \times[-2,0]$. Here $u$ wave contour is maximum on the top of the wave but diminishes from the top to both the sides of contour. At the top the value of the contour is -283.903 and it gradually decreases to the both sides like -300 to the left and -300 to the right.

\subsection{Case II}

For the second case: $\lambda^{2}-4 \mu=0$

$$
\begin{gathered}
G(\xi)=\left(c_{1}+c_{2} \xi\right) \exp (-\lambda \xi / 2), \\
\therefore G^{\prime}(\xi)=\frac{-\lambda c_{1}+c_{2}(2-\lambda \xi)}{2} \exp (-\lambda \xi / 2)
\end{gathered}
$$

Therefore, $\frac{G}{G^{\prime}}=\frac{2(1+c \xi)}{-\lambda+c(2-\lambda \xi)}$

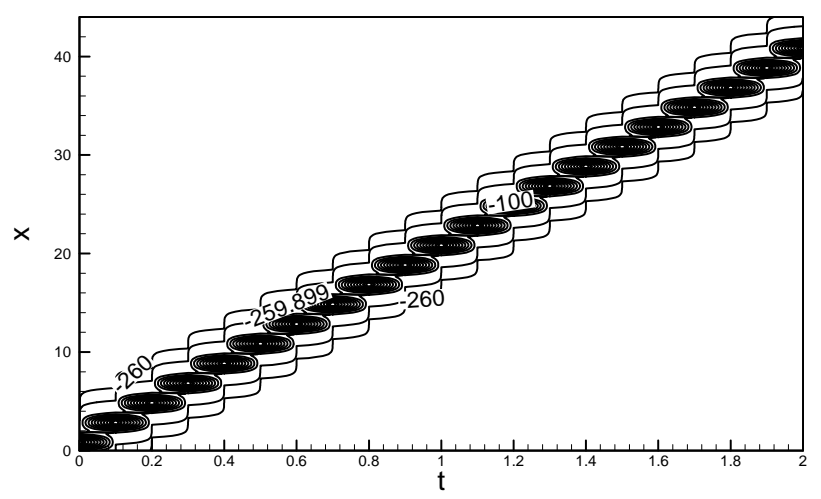

Figure 8. $u$ wave contour against $t$ and $x$.

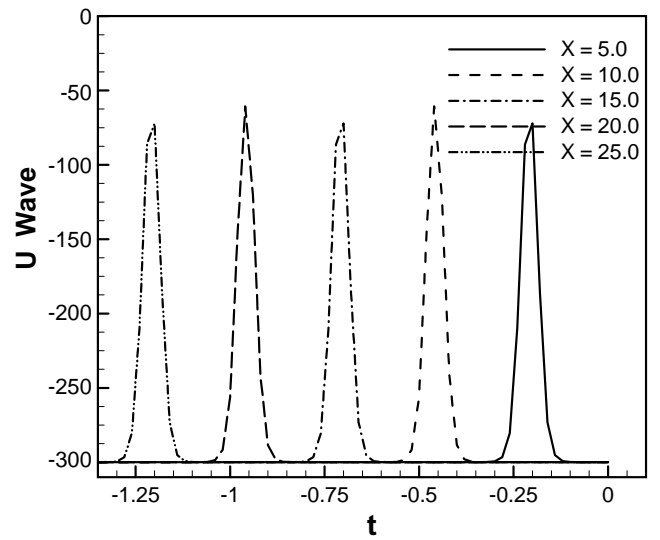

(a)

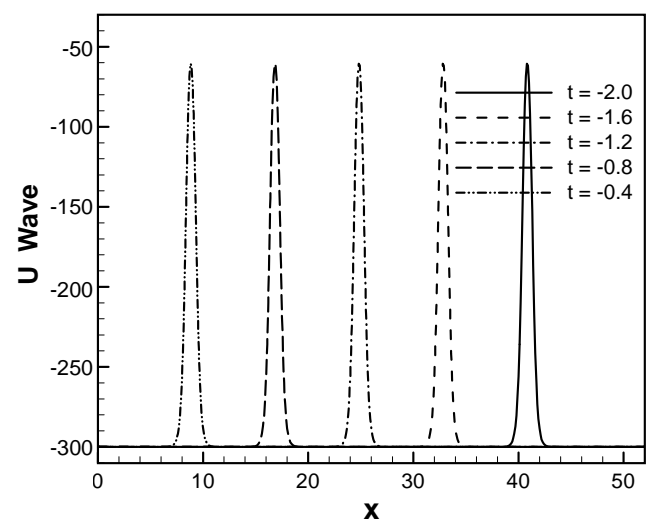

(b)

Figure 9. $u$ wave (a) against $t$ for different values of $x$ (b) against $x$ for different values of $t$.

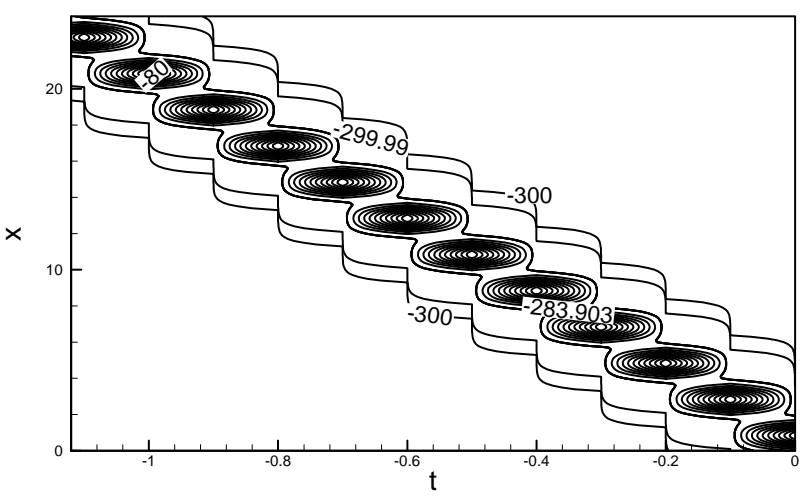

Figure 10. $u$ wave contour against $t$ and $x$.

Equations (F)-(J) and (10) implies respectively as follows:

$$
\begin{aligned}
& u(\xi)=-24 \lambda \mu\left[\frac{1+c \xi}{-\lambda+c(2-\lambda \xi)}\right] \\
&-48 \mu^{2}\left[\frac{1+c \xi}{-\lambda+c(2-\lambda \xi)}\right]^{2} ; \\
& \xi=x-\left(\lambda^{2}+8 \mu\right) t
\end{aligned}
$$




$$
\begin{aligned}
& u(\xi)=-12 \mu-6 \lambda\left[\frac{-\lambda+c(2-\lambda \xi)}{1+c \xi}\right] \\
& \quad-3\left[\frac{-\lambda+c(2-\lambda \xi)}{1+c \xi}\right]^{2}, \\
& u(\xi)=-2 \lambda^{2}-4 \mu-6 \lambda\left[\frac{-\lambda+c(2-\lambda \xi)}{1+c \xi}\right] \\
& \quad-3\left[\frac{-\lambda+c(2-\lambda \xi)}{1+c \xi}\right]^{2}, \\
& \xi=x \quad]^{2} \\
& u(\xi)=8 \mu-12 \mu^{2}\left[\frac{1+c \xi}{c}\right]^{2}-12\left[\frac{c}{1+c \xi}\right]^{2} \\
& \xi=x-16 \mu t, \lambda=0 \\
& \lambda=0, \Rightarrow \mu=0 \text { then, } \\
& u(\xi)=-12\left[\frac{c}{1+c \xi}\right]^{2} ; \xi=x
\end{aligned}
$$

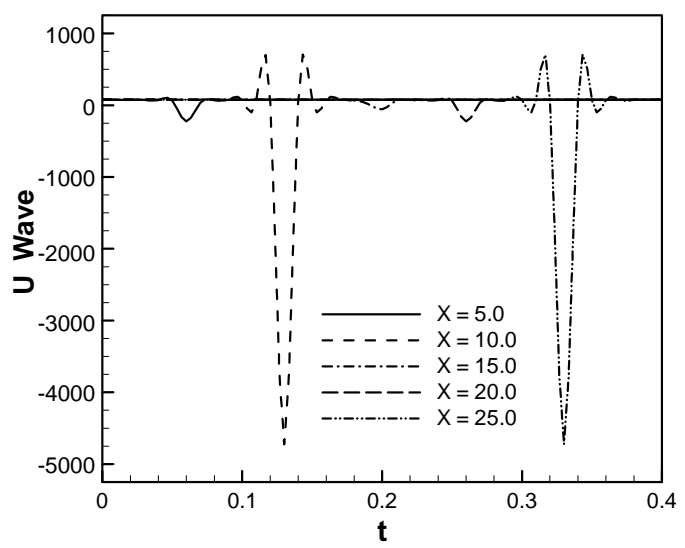

(a)

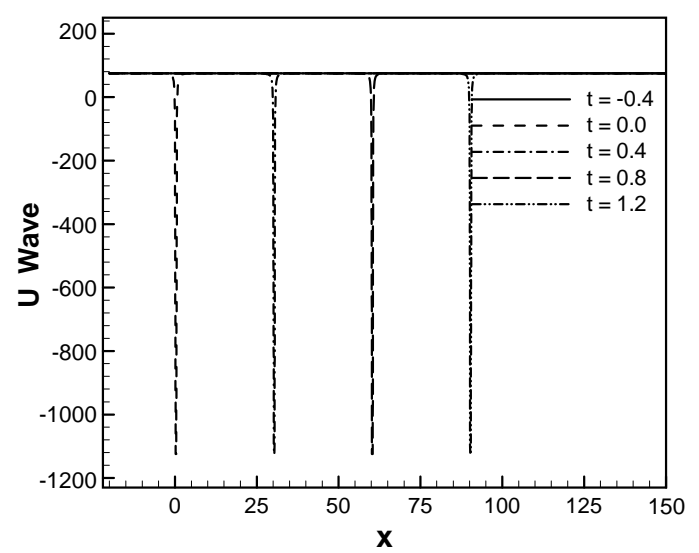

(b)

$$
\begin{aligned}
& u(\xi)=-24 \mu-12 \mu^{2}\left[\frac{1+c \xi}{c}\right]^{2}-12\left[\frac{c}{1+c \xi}\right]^{2} ; \\
& \xi=x+16 \mu t, \lambda=0
\end{aligned}
$$

$u(\xi)=-12\left[\frac{c_{3}}{1+c_{3} \xi}\right]^{2} ; \xi=x$ which is same as Equation (20).

The numerical representation of two dimensional third order $\mathrm{K}-\mathrm{dV}$ equation for this problem is obtained by the Fortran scheme compared with analytical solution to the following cases for $\lambda=5.0, \mu=6.25$ and $c=12.0$ such that $\lambda^{2}-4 \mu=0$ in the real sense. Numerical solution generates that the same behavior as solitary wave solutions for different cases. The solutions remain unchanged before and after their intersection. As seen in Figures 11(a) and (a1) for the Equation (17), time evaluation of $u$ wave for different values of displacement like $x=5.0, x=10.0, x=15.0, x=20.0$ and $x=25.0$. For a particular value of $x=10.0, u$ wave simply solitary. But the second case Figures 11(b) and (b1) for the same equation, u-wave generates solitary for different values of $t=-0.4, t=0.0, t=0.4, t=0.8$ and $t=1.2$. Here

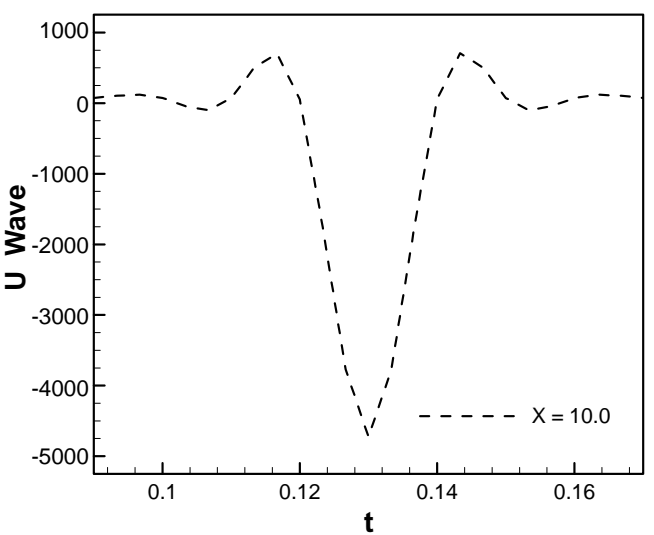

(a1)

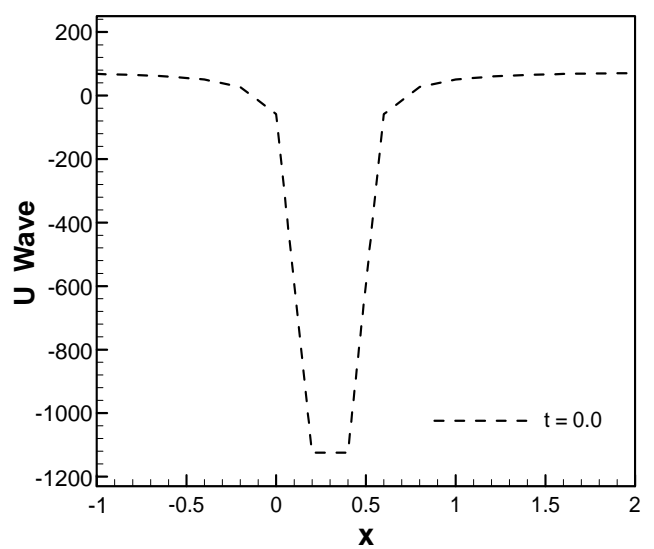

(b1)

Figure 11. $u$ wave (a) against $t$ for different values of $x(b)$ against $x$ for different values of $t$. 
negative sign of $t$ indicates before the collision. For a particular value of $t=0.0$ the $u$ wave is solitary. In Figure 12 shows that the contour is produced against $t$ and $x$ simultaneously. In this equation we get the numerical representation of the solution of Equation (18) for $\lambda=5.0, \mu=6.25$ and $c=12.0$ such that $\lambda^{2}-4 \mu=0$. But for the values $c=10.0, c=12.0, c=15.0, c=17.0$ we observe that $u$ wave is solitary. For a particular value of $c=12.0, u$ wave generates solitary which is represented in Figure 13(b). Next, contours of typical flow pattern are seen in Figures 14(a) and (b) which is based on Equation (19). The waves associate over the interval $-3 \leq x \leq 3$. It is found that the flow oscillates regularly. We are also interested in trying our scheme for the wave solution of the $\mathrm{K}-\mathrm{dV}$ equation. In this figure we have seen that the behavior of $u$ wave is exactly that what we expect in the analytical sense, the only thing is that the

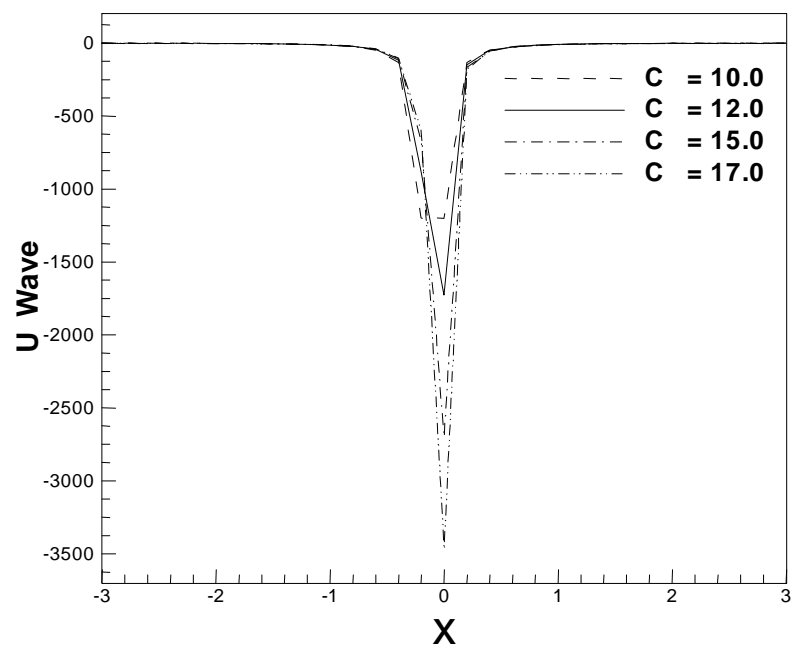

(a) change about the wave once for the single value of $c$. For Figure 14(a) the oscillation is no doubt solitary before and after the collision for different values of $c=10.0, c$

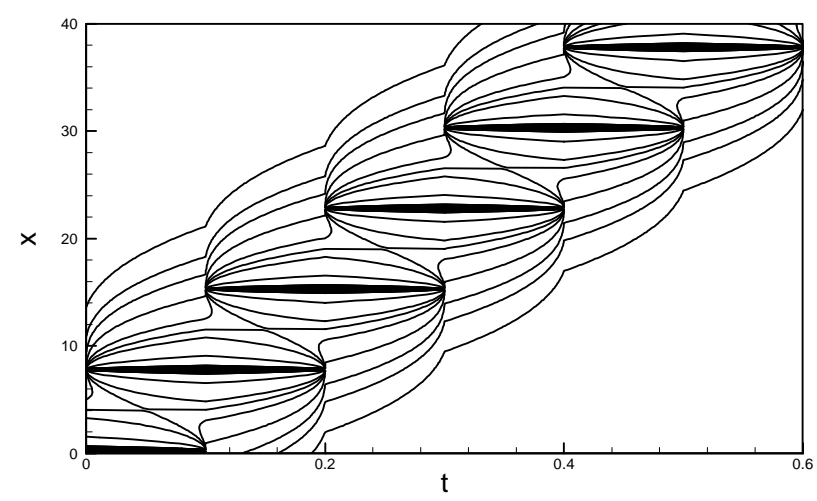

Figure 12. $u$ wave contour against $t$ and $x$.

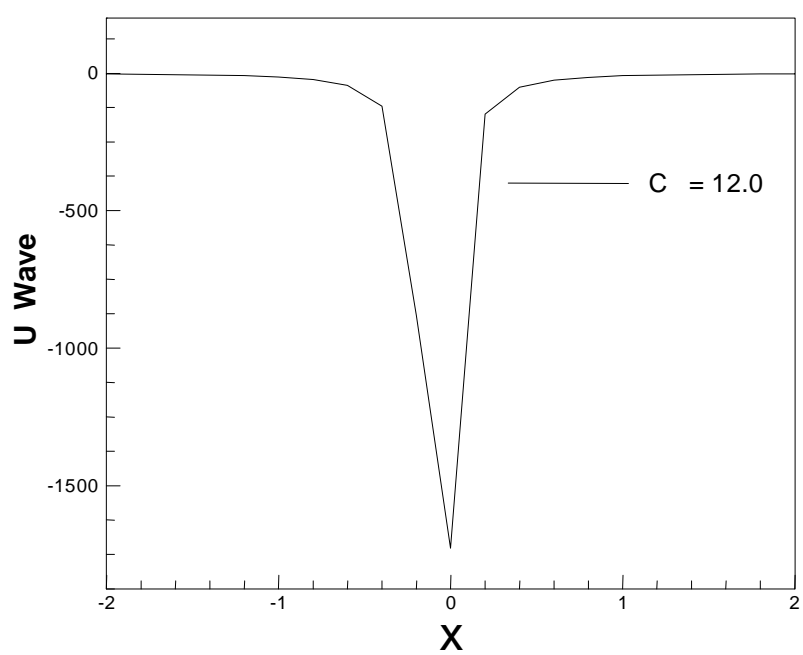

(b)

Figure 13. $u$ wave against $x$ for different values of $c$.

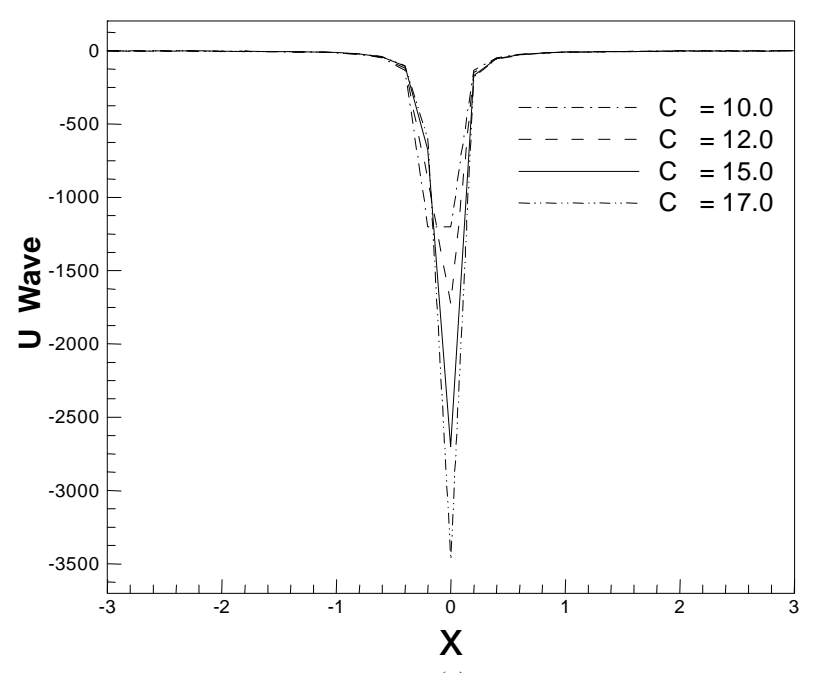

(a)

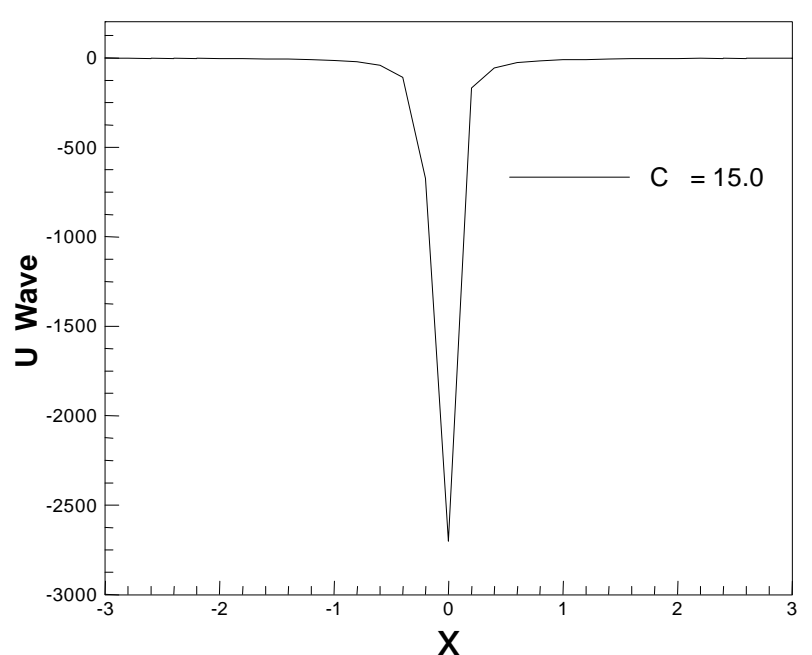

(b)

Figure 14. $u$ wave against $x$ for different values of $c$. 


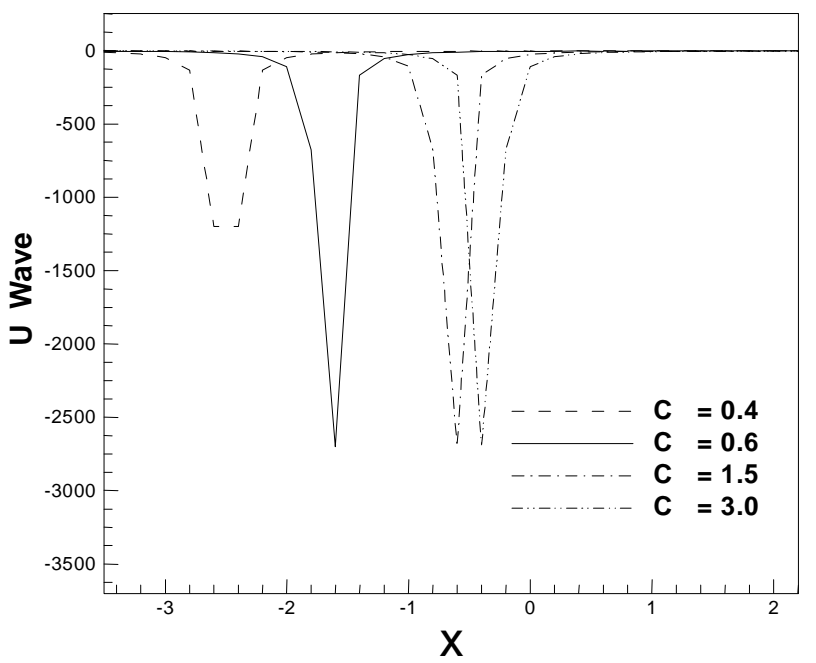

(a)

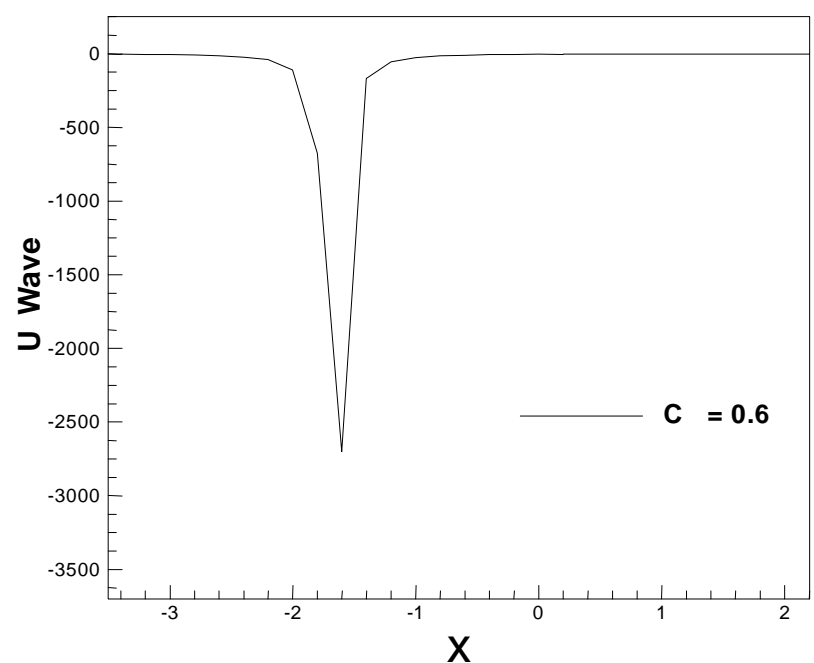

(b)

Figure 15. $u$ wave against $x$ for different values of $c$.

$=12.0, c=15.0, c=17.0$. But in Figure 14(b) for a particular value of $c=15.0$, the wave is very clear. The plot of Equation (20) is shown in Figures 15(a) and (b). We have found wave against for different values of $c$. The waves against $x$ for different values of $c=0.4, c=0.6, c$ $=1.5$, and $c=3.0$ for $\lambda=5.0, \mu=6.25$, and $c=12.0$ such that $\lambda^{2}-4 \mu=0$ are exact that we have got in the analytical sense for the Equation (20). But in Figure 15(b) represents a solitary wave against $x$ for a particular value of $c$.

\section{Conclusion}

In this research numerical estimation of traveling wave solution for third order of two-dimensional K-dV equation using a new auxiliary equation method has been studied. The $\mathrm{K}-\mathrm{dV}$ equation for the present problem comes from the third order two dimensional governing Equation (1.1) after some suitable transformation. It is found that there are nine exact traveling wave solutions (12)-(20) of 2-dimentional K-dV equation exist for real sense depends on different relevant physical parameters but the last one is exact the same as (20). Numerical results of first ten cases for real sense obtained by using FORTRAN program have been shown graphically and discussed. We have also found that when employing the Fortran-Scheme for the numerical estimation of K-dV equation that are presented graphically for the first case $\lambda^{2}-4 \mu>0$ and second case $\lambda^{2}-4 \mu>0$ Equations (12)(20). Remaining imaginary cases will be avenue of another research work in the future.

\section{REFERENCES}

[1] Yu. N. Zaiko, "Quasiperiodic Solutions of the Kortewegde Vries Equation," Technical Physics Letters, Vol. 28,
No. 3, 2002, pp. 235-236. doi:10.1134/1.1467286

[2] F. L. Qu and W. Q. Wang, "Alternating Segment Explicit-Implicit Scheme for Nonlinear Third-Order KdV Equation," Applied Mathematics and Mechanics, Vol. 28, No. 7, 2007, pp. 973-980. doi:10.1007/s10483-007-0714-y

[3] N. E. Zhukovskii, "Hydraulic Shock in water Pipelines," Gostekhteorizdal, Moscow, 1949.

[4] A. C. Newell, "Solitons in Mathematics and Physics," SIAM, Philadelphia, 1985.

[5] V. A. Rukavishnikov and O. P. Tkachenko, "The Korteweg-de Vries Equation in a Cylindrical Pipe," Computational Mathematics and Mathematical Physics, Vol. 48, No. 1, 2008, pp. 139-146. doi:10.1134/S0965542508010107

[6] N. Smaoui and R. H. Al-Jamal, "Boundary Control of the Generalized Korteweg-de Vries-Burgers Equation," Nonlinear Dynamics, Vol. 51, 2008, pp. 439-446.

[7] J. Pang, C.-Q. Bian and L. Chao, "A New Auxiliary Equation Method for Finding Traveling Wave Solution to KdV Equation," Applied Mathematics and Mechanics (English Edition), Vol. 31, No. 7, 2010, pp. 929-936.

[8] L. Debnath, "Linear Partial Differential Equations for Scientists and Engineers," 4th Edition, Tyn Myint-U, 2007, pp. 573-580.

[9] R. L. Herman, "Solitary Waves," American Scientist, Vol. 80, 1992, pp. 350-361.

[10] P. A. Clarkson and M. D. Kruskal, "New Similarity Reductions of the Boussinesq Equation," Journal of Mathematical Physics, Vol. 30, No. 10, 1989, pp. 2201-2213. doi: $10.1063 / 1.528613$ 